\title{
400-Euro-Jobs richtig dokumentieren
}

- Bei mehreren parallel ausgeübten Minijobs kann durch Überschreiten der monatlichen Entgeltgrenze von 400 Euro eine Versicherungspflicht eintreten. Um die versicherungsrechtliche Beurteilung der Beschäftigung richtig durchführen zu können, sollte der Arzt als Arbeitgeber daher bei Beginn einer solchen Beschäftigung abfragen, ob der betreffende Arbeitnehmer - z.B. die Reinigungskraft - bereits bei anderen Arbeitgebern geringfügig oder versicherungspflichtig beschäftigt ist.

Es empfiehlt sich, einen „Personalfragebogen für geringfügig Beschäftigte" auszufüllen und vom Arbeitnehmer unterschreiben zu lassen.

\section{MMW Kommentar}

Wenn ein Sozialversicherungsträger erst im Nachhinein zum Beispiel durch Datenabgleich oder im Rahmen einer Betriebsprüfung, feststellt, dass die Beschäftigung vom Arbeitgeber falsch beurteilt wurde und eigentlich keine Geringfügigkeit und damit Versicherungsfreiheit besteht, tritt diese erst mit der Bekanntgabe der Feststellung durch die Einzugsstelle oder durch einen Rentenversicherungsträger ein. Sie gilt damit nur für die Zukunft, während für die zurückliegende Zeit die Beschäftigung ver- sicherungsfrei bleibt. Diese Regelung gilt allerdings nicht, wenn der Arbeitgeber vorsätzlich oder grob fahrlässig versäumt hat, den Sachverhalt für die versicherungsrechtliche Beurteilung aufzuklären. Dies kann unterstellt werden, wenn der Arbeitgeber den Arbeitnehmer bei Beschäftigungsbeginn nicht nach weiteren Beschäftigungen gefragt bzw. die Angabe weiterer Beschäftigungen ignoriert hat. In diesem Fall tritt eine Versicherungspflicht auch für die Vergangenheit ein und die Beiträge müssen nachgezahlt werden!

\section{Praxisbesonderheiten gezielt melden}

- Im Arznei- und Heilmittelbereich werden Wirtschaftlichkeitsprüfungen auf der Basis der Richtgrößen bzw. des Richtgrößenvolumens durchgeführt. Überschreitet eine Praxis ihr Richtgrößenvolumen bei den Arznei- oder Heilmitteln eines gesamten Jahres um mehr als $25 \%$, wird eine Richtgrößenprüfung eingeleitet. Führen Praxisbesonderheiten, wie beispielsweise sehr teure Behandlungsfälle nicht zu einer Reduzierung des Überschreitungsvolumens auf unter $25 \%$, droht ein Richtgrößenregress.

Aus diesem Grund können von niedergelassenen Vertragsärzten Praxisbesonderheiten als „vorsorgliche Meldungen“ an die Prüfgremien weitergeleitet werden. Oft ist ein solcher Vorabaufwand aber nicht notwendig, da die Prüfstellen die Möglichkeit haben, die Verordnungen im Arznei- und Heilmittelbereich der Ärzte über eine Datenbank einzusehen und so Praxisbesonderheiten selbst zu erkennen. Diese Vorabprüfung führt dazu, dass mehr als $80 \%$ der Prüfanträge der Krankenkassen hinfällig werden, da besonde- re Verordnungen die Richtgrößenüberschreitung erklären bzw. auf unter $25 \%$ reduzieren. Zeigen die Verordnungen in der Vorabprüfung für die Prüfstelle keine Praxisbesonderheiten, und wird die Richtgrößenüberschreitung von mehr als $25 \%$ entsprechend reduziert, informiert die Prüfstelle die Praxen und bittet, ggf. vorhandene Praxisbesonderheiten bei der Verordnung zu melden. Dies führt meist dazu, dass auch dann noch in mehr als 50\% dieser Fälle vorhandene Praxisbesonderheiten die Richtgrößenüberschreitung erklären und kein Regress resultiert.

\section{MMW Kommentar}

So gesehen reicht es aus, Praxisbesonderheiten möglichst genau zu dokumentieren, aber keine „vorsorglichen Meldungen über Praxisbesonderheiten " zu verschicken. Denn erst dann, wenn ein Richtgrößenverfahren eingeleitet wird, fragt die Prüfstelle danach und die betroffene Praxis kann mit einer Stellungnahme gezielt die vorhandenen Praxisbesonderheiten darlegen.

\section{Werbeverbot gelockert}

- Nach einem Urteil des Bundesverwaltungsgerichts darf ein Arzt mit Leistungen, die über dem Standard der gesetzlichen Krankenkassen liegen, werben. Hinweise dieser Art sind nach Auffassung der Richter eine angemessene Information des Publikums. Ein Verbot ist deshalb mit dem Grundrecht der freien Berufsausübung nicht vereinbar (Az.: $3 C_{4}$ /og).

\section{MMW Kommentar}

Der konkrete Fall bezieht sich zwar auf eine Gruppe von etwa 40 Zahnärzten bundesweit, kann aber auf den humanmedizinischen Bereich übertragen werden. Die Gruppe hatte sich unter einem Namen zusammengeschlossen und aus eigenen Stücken zu höheren Behandlungsstandards verpflichtet. Dabei setzten sie ein Qualitätssiegel ein, um Patienten auf das Angebot aufmerksam zu machen. Die zuständige Zahnärztekammer untersagte dies, scheiterte aber nun vor dem Bundesverwaltungsgericht. 